\title{
Cautious welcome to NIH peer review reforms
}

Washington. Encouraged by preliminary results, the US National Institutes of Health (NIH) are to expand an experiment in modifying the peer-review system for research grants. The goal is to reduce the time spent dealing with applications that are clearly not likely to win a grant, and to increase that spent evaluating borderline cases.

Nearly 80 per cent of the reviewers who took part in an initial small trial said that they had the same level of confidence in their recommendations as under the current system. Almost 60 per cent said they would approve the new approach if some additional modifications were made.

The approach is known as 'triage', after the military procedure for sorting battlefield casualties into priorities for treatment. Jerome Green, director of NIH's Division for Research Grants (DRG), says that a decision on whether to extend it to the whole of NIH is likely to be made at the end of the year.

There are several reasons behind NIH's desire to modify its peer review procedures. One is to speed up the process; at present, grant applicants have to wait nine months to hear whether they have been successful, and most are disappointed. The NIH can fund fewer than a quarter of the applications it

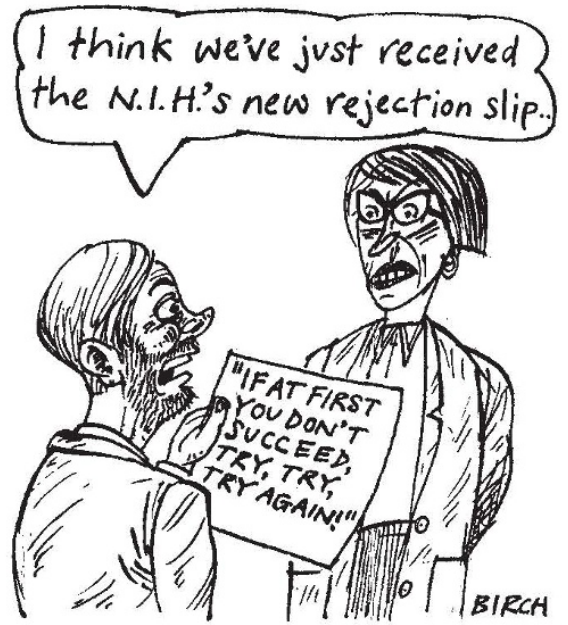

receives, and reviewers therefore spend much unpaid time evaluating applications that will be unsuccessful anyway. This has created a sense of frustration in the scientific community, making it difficult for the NIH to recruit reviewers.

Unsolicited proposals are the bedrock of biomedical research in the United States, and account for nearly half the NIH's extramural research funding ( $\$ 8.5$ billion in the financial year 1993). The triage approach focuses on the first of two stages in the review process, that concerned with a purely scientific review of applications. The second involves evaluating applications that pass the first stage against the research and other priorities of the individual institutes.

At present, the DRG assigns all unsolic- ited proposals either to a study section within the division, or to a panel within an individual institute, each made up of 15 to 20 senior scientists or specialists in the field.

Before the panel or section meets, the NIH scientist responsible for its work sends every application to two reviewers. These assign a score to the application ranging from 500 (lowest) to 100 (highest), based on factors such as its significance, the appropriateness of methodology, the qualifications of investigators and the resources available in the investigators' home institution.

The scores are passed to the DRG, where they are adjusted to compensate for differences between study section members. The panel then discusses all applications, eventually deciding not to recommend some for further consideration. An NIH staff member then sends unsuccessful applicants a summary of reviewers' criticisms and remarks emerging from the panel discussion.

The applications that survive their scientific peers are sent to the second level of review within the individual institutes, and eventually about 20 per cent of the applications are funded. In general, successful applications have both the lowest scores from the scientific reviewers and are ranked above the fourteenth or fifteenth percentile or so of the overall range of scores.

Triage aims to identify as "noncompetitive' applications below the fiftieth percentile. If both initial reviewers agree with this assessment, and no other scientific reviewer disagrees, the application is not discussed further and the NIH staff member does not write a summary of the reasons for rejection.

The applicant does, however, receive the criticisms of the two reviewers, thus maintaining most of the tutorial element of peer review. Of the four (out of over a hundred) study sections involved in the trial so far, only the Human Development and Aging Study Section winnowed out all those below the fiftieth percentile, which in that case constituted 51.1 per cent of applications.

The new approach has met with a generally favourable response. Keith Yamamoto, chair of the department of pharmacology at the University of California, San Francisco, who is organizing a seminar on peer review at the NIH in the autumn, says that "on balance" he is in favour of triage: "It gives more time to discuss the top proposals."

At the same time, says Yamamoto, "there is a real danger that innovative research will be triaged out". This concern stems from a suspicion that reviewers, under the pressure of tight budgets, are becoming increasingly conservative, and now favour proposals backed by significant preliminary data.

"I can't tell you how often I have read a review that says this proposal has the potential to advance the field tremendously, but it is high risk," says Yamamoto. "In fact, I've written that a few times myself."

Yamamoto believes that, in order to preserve high-risk, high pay-off science, the instructions to reviewers should explicitly require them to consider innovativeness along with the other criteria. "What has made American science so enterprising is the ability of some people to move nonlinearly," he says. "We must not cut these people out of the loop."

Others are concerned that eliminating the detailed assessments of applications that unsuccessful applicants receive will remove a valuable service to the scientific community, as they are essentially a free consultation with leading scientists in a field.

Green says he his aware of this, but it needs to be put in perspective. "We need to balance the need to shorten the time that scientists and NIH staff spend on each application against the importance of maintaining the tutorial aspect of peer review," he says.

Although the triage trial is to be expanded, a significant reduction in the time it takes for applicants to hear their fate is likely to come only with a move to electronic filing and processing of grant applications. "At present, we are in the ridiculous position where the researchers will have written up their application on computer, yet we receive them and key them in, then print out numerous hard copies," says Green. Tight funding means that full implementation of electronic processing will probably take at least five years.

Helen Gavaghan

\section{Brussels boost to UK universities}

London. The research income received by British universities from the European Commission (EC) in Brussels increased by a hefty 32 per cent last year to reach a total of $£ 76$ million - 7.0 per cent of their total research funding - according to figures published last week by the Universities Statistical Record (USR).

In contrast, research grants from private industry rose by a meagre 0.8 per cent, to $£ 121$ million. This compares to an increase of 5.3 per cent in the previous year, and is "probably due to the recession", says the USR's University Statistics 1992-93, Volume 3: Finance.

Some universities did particularly well in boosting their income from EC sources. The University of Cambridge, for example, which has been protesting strongly about having its domestic funds "capped" by the government in order to maintain support for less productive institutions, saw its EC income almost double, to $£ 3.8$ million. 